\title{
A smart login system using face detection and recognition by ORB algorithm
}

\author{
Mohammad Jahangir Alam, TanjiaChowdhury’Md. ShahzahanAli \\ Department of Computer Science and IT, Southern University Bangladesh, Bangladesh
}

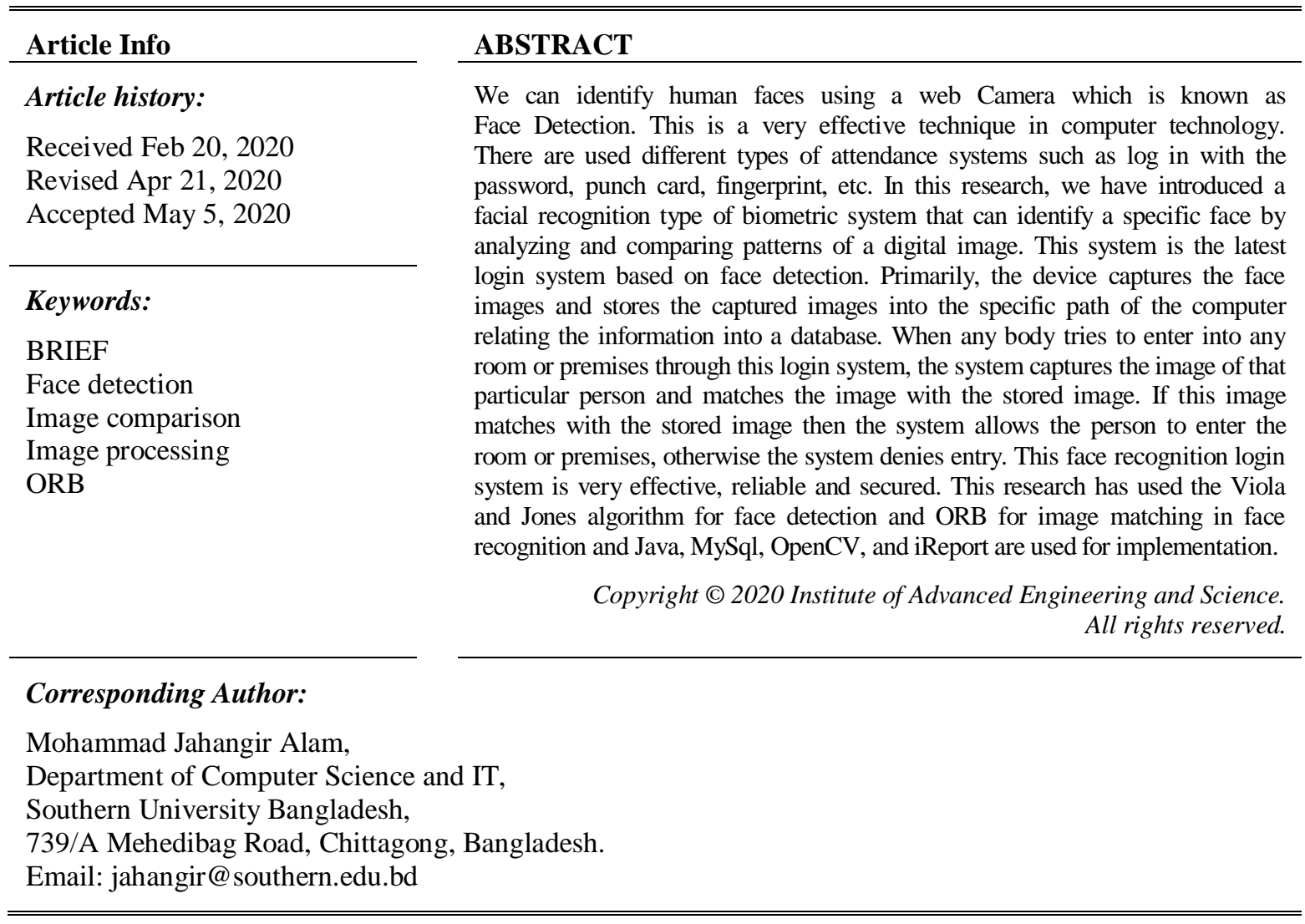

\section{INTRODUCTION}

In our daily lives, we often remember and recognize people by looking at their faces. This is a part of the body that is highly visible and is important for interaction. We store information of a face and later use that information for recognition and matching purposes. This mechanism can be used by machines to recognize and authenticate a human being with the increasing importance of technology in business and human lives where security has become a critical concern for modern applications. Users' authentication is the most important part of securing an application from unauthorized access. For this, knowledge-based, token-based and biometric based systems can be used. Traditional knowledge-based and token-based systems are losing appeal due to the issues associated with their usage. This situation has increased the importance of biometric (what we are) characteristics rather than knowledge (what we know) and token (what we have) approaches [1]. In our country there many types of login systems are used such as login by punch card, user id or password. But in these systems, there is possibility of misusing the systems through mutual understanding. The developed smart system can overcome the previously developed systems' limitations. The system checks individual user's face with existing stored faces, that's why there is no chance of misuse.

\section{Objective}

a) To help organizations to make their login systems more secured and smart.

b) To detect individuals by using the face detection system. 
c) To reduce the use of punch cards or any common login systems.

d) To generate Automatic login report.

e) To monitor easily.

\section{LITERATURE REVIEW}

Here, we have illustrated some related works which have been conducted by the researches previously. We have studied the research works and tried to find out the limitations of the researches. Such as Yogesh Maniktala et al. proposed a Robust Technique of Face Recognition [2]. In this research, they have described the face detection process and recognition systems using pixels and analyzing the images. Smriti Tikoo et al. developed a Face detection System using Viola Jones and Recognition system using Back Propagation Neural Network [3]. In this paper, the facial detection has been carried out using the Viola-Jones algorithm and recognition of face has been done using Back Propagation Neural Network (BPNN). Dr. Nita Thakare et al. have carried research on "Face Detection and Recognition for Automatic Attendance System" [4]. In this research, they have described their experience in developing face detection and recognition system for attendance software. Gunjan Mehta et al. was introduced a Face Recognition System using PCA, FLDA and Artificial Neural Networks [5]. The paper presented a technique to implement a system that aims to describe four different methodologies for Face Recognition. There is another paper where neural a network is also used for detecting a face. This paper proposed a face localization technique and developed a new feature extraction algorithm for human face recognition. There is another paper where the neural network is also used for detecting a face. This paper proposed a face localization technique and developed a feature extraction algorithm, Neural Network (NN) using Back Propagation Networks (BPN) and Radial Basis Function $(\mathrm{RBF})$ networks for human face recognition. The neural network model is used for recognizing the frontal or nearly frontal faces and the results are tabulated [6]. Mamata et al. developed a real-time face detection and tracking using OpenCV [7]. In this paper, they represented a methodology for face detection in a real-time environment and was used Harr like classifier and AdaBoost algorithm for tracking faces on the OpenCV platform which is an open source and developed by Intel. D. A. R. Wati et al. also used Face detection and recognition systems for smart home security applications [8]. To implement this system, they used MyRIO 1900 and LabVIEW for programming. For performance evaluation, they have considered several variables like a change of distance, light intensity, light position angles, person's fixtures, and shirt color. To increase the system security they added the password field. Aanjana Devi. S et al. were proposed a confidential e-voting system using face detection and recognition. Eigenface algorithm and viola jones were used for implementation which provides high security, and reliability [9]. Haar-Like feature and Eigenfaces also applied as an algorithm for detecting the face of a visually impaired person. In this paper, researchers developed a smart cane for recognition of face by using Haar-like features and Eigenfaces [10]. There is another paper, Facial Action Coding System (FACS), applied for detecting facial expression. They worked with six basic emotions of happiness, sadness, shock, fear, anger, and disgust [11]. Reivind P. Persada et al. proposed a security system on Smart Parking based on face recognition and VLP's (Vehicle License Plates) identification. In this paper, the Structural Similarity (SSIM) method applied for reliable and simple computation for the face detection and recognition process [12]. Ghazal et al. research on Fast Discrete Curvelet Transform (FDCvT) and Invariant Moments with Support vector machine (SVM), these two algorithms are used for face recognition [13]. J. Vinoj et al. research to provide a security system in the smart city through the vehicle login system, the researcher developed a sensor chip-based model [14]. Kumar et al. the researchers proposed a review paper based on face detection techniques [15]. There are many researchers used face recognition and detection system in various purposes; even they developed a security system for face recognition [16-21]. From the above-mentioned research works, we have had some idea relevant to the system and conducted this research to overcome the previous limitations and to introduce the new idea.

\section{THE METHOD OF SYSTEM DESIGN}

Image processing is a smart and modern technology. We have studied various methods of image processing. In this section, we described the algorithm of face detection and recognition. We have used the Viola and Jones algorithm for face detection and ORB (Oriented FAST and Rotated BRIEF) for image matching in face recognition. The BRIEF algorithm works as descriptor and FAST works as a detector and ORB works as both descriptor and detector for image matching. The overview of all algorithms is briefly described below:

\subsection{Viola and Jones algorithm for face detection}

The Viola and Jones algorithm is a good detection algorithm because of its robustness. A vital contribution of the method is the use of the integral image representation together with Haar-like features, 
shown in Figure 1. There are four types of features, the output at a specific coordinate position is a scalar value calculated from the difference between grey and white areas of the feature. The values of the gray and white areas are determined by the sums of pixels values within. The calculation of all features at a certain position is thus a succession of double sums (in $\mathrm{x}$ and $\mathrm{y}$ direction) and subtractions.

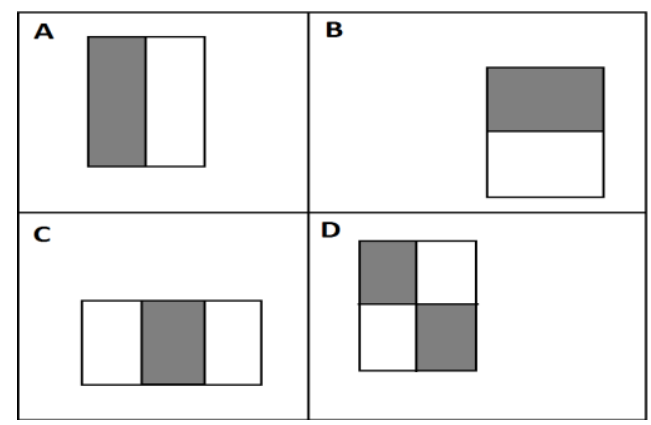

Figure 1. The four types of Haar-like features used in the Viola-Jones method

The integral image, also known as a summed-area table, is a technique that enables fast and simple computations of sums over rectangular areas in an image. Each pixel (x,y) in the integral image is equal to the sum of pixels above and to the left of $(x ; y)$ in the original image, as shown in (1).

$$
\text { I I }(x, y)=\underset{x^{\prime} \leq x, y^{\prime} \leq y}{\sum}\left(x^{\prime}, y^{\prime}\right)
$$

Any rectangular sum can then be calculated using very few operations. In practical terms, an integral image is the double cumulative sum of an image, along the row dimension and the column dimension. Each point in the integral image corresponds to the sum of the original image up to that point, as Figure 2.

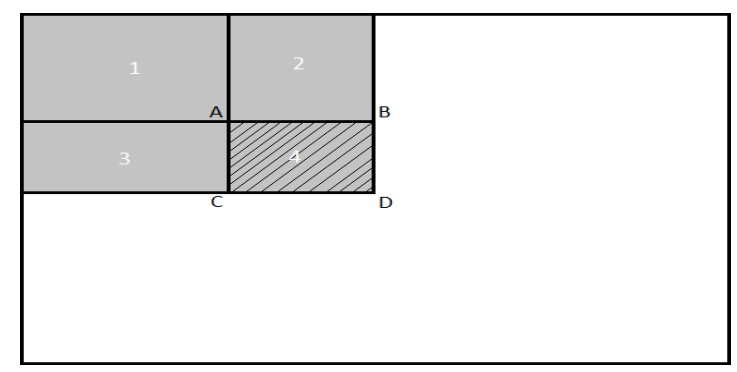

Figure 2. The sum of the gray area in the image corresponds to point $\mathrm{D}$ in the integral image

Any arbitrary rectangle can be defined in the image as four points, and these points are the four corners of the rectangle, A, B, C and D as shown in Figure 2. There are also four areas numbered in the Figure, and we can now define the value each of these four corners as a sum of these areas. Point A in the integral image corresponds to area $1, \mathrm{~B}$ is the union of 1 and $2, \mathrm{C}$ is the union of 1 and 3 and $\mathrm{D}$ is the union of all gray areas. This gives us

$$
\text { Area } 4=\mathrm{II}(\mathrm{D})+\mathrm{II}(\mathrm{A})-\mathrm{II}(\mathrm{C})-\mathrm{II}(\mathrm{B})
$$

\section{The algorithm has four stages:}

a) Haar Feature Selection

b) Creating an Integral Image

c) Adaboost Training

d) Cascading Classifiers [10]. 


\subsection{Binary robust independent elementary features (BRIEF)}

BRIEF is a feature descriptor. It provides a shortcut to find the binary strings directly without finding descriptors. It takes a smoothened image patch and selects a set of $(\mathrm{x}, \mathrm{y})$ location pairs uniquely. Then some pixel intensity comparisons are done on these location pairs. For example, let the first location pairs $I(p)<I(q)$, then its result is 1 , else it is 0 . This is applied for all the $n_{d}$ location pairs to get a $n_{d}$ dimensional bi string. This $n_{d}$ can be 128,256 or 512 . OpenCV supports all of these, but by default, it would be 256 (OpenCV represents it in bytes. So the values will be 16, 32 and 64). So, once you get this, you can use Hamming Distance to match these descriptors [22].

\subsection{FAST algorithm for corner detection}

A FAST algorithm is used for image matching. This algorithm works with three steps namely-

Feature Detection using FAST ii) Machine Learning a corner detector and iii) Non-maximal suppression.

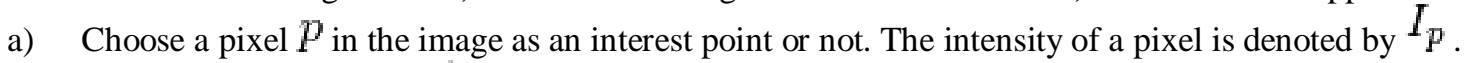

b) Choose a threshold value $t$.

c) Select a circle of $\mathrm{n}$ pixels around the pixel under test.

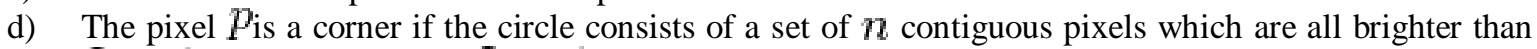
$I_{p}+t$, or all darker than $I_{p}-t$. In below Figure 3, there are 16 pixelsshown as white dash lines and for $\mathrm{n}=12$.

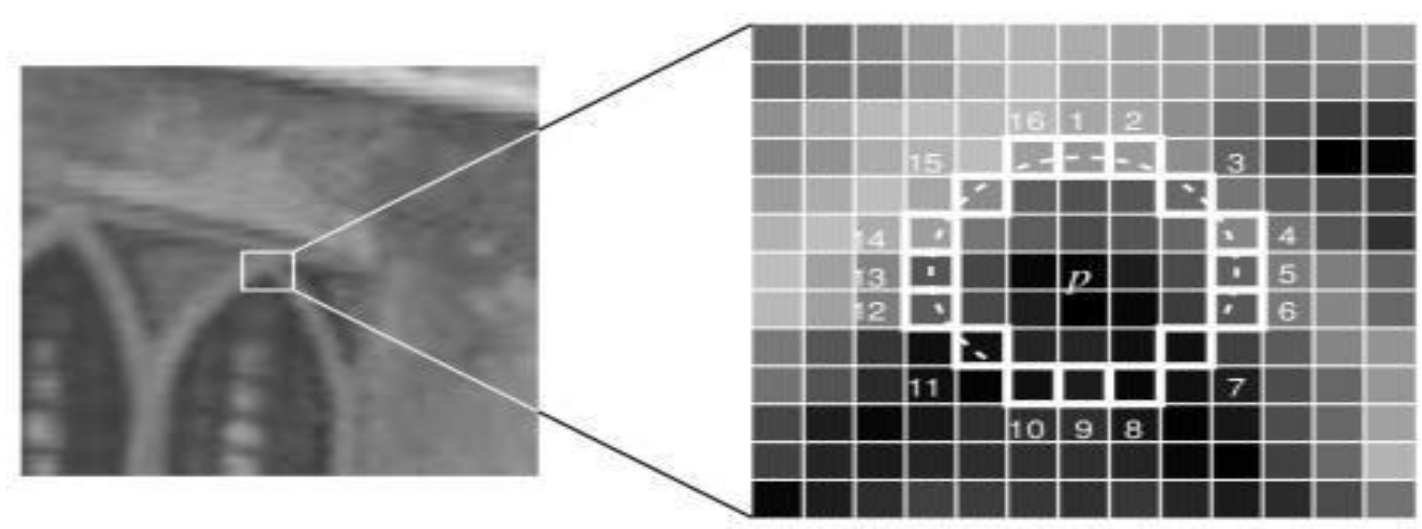

Figure 3. Contiguous pixel

e) A high-speed test was proposed to eliminate a large number of non-corners. If $p$ is a corner, then the pixels must all be brighter than $I_{p}+t$ or darker than $I_{p}-t$. If not, then $p$ cannot be a corner. In the test, the four pixels at $1,9,5$, and 13 are examined. Here, First 1 and 9 are tested if they are too brighter or darker. If so, then checks 5 and 13 which shown in the above Figure 3 . This detector provides high performance, but there is some lacking which are:

a) It does not eliminate many candidates.

b) The choice of pixels is not optimal.

c) Results of high-speed tests are thrown away.

d) Multiple features are detected adjacent to one another.

The first 3 points are addressed with a machine learning approach. The Last one is addressed using non-maximal suppression [23, 24].

\subsection{Oriented FAST and rotated BRIEF (ORB)}

ORB is a modified version of the FAST detector and BRIEF descriptor which increases the performance. It calculates the intensity weighted centroid of the patch with the located corner at the center. The performance of the BRIEF is poor.Instead of ORB use BRIEF descriptors. ORB is to "steer" BRIEF according to the orientation of key points. For any feature, we consider a set of $n$ binary tests at the location $\left(x_{i}, y_{i}\right)$, define a $2 \times n$ matrix, and $S$ is the coordinates of these pixels. By applying the orientation of patch, $\theta$, its rotation matrix is found and rotates the $S$ to get steered(rotated) version $S_{\theta}$. OR Bincrements the angle of $2 \pi / 30$ (12 degrees), and build a lookup table of recomputed BRIEF patterns. If the key point orientation $\theta$ is consistent across assessments, the correct set of points $S_{\theta}$ will be used to 
compute its descriptor. BRIEF provides high variance and a means near 0.5. When it works with key point direction, it loses high variance and means which make it distributive. A feature becomes more discriminative because of high variance. To overcome these problems, ORB uses a greedy search among all possible binary tests to achieve high variance and means close to 0.5 , as well as being uncorrelated which is known as BRIEF [22]. Using the below Figure 4, we have calculated the orientation of the patch; rotate it to a canonical rotation, we can compute the descriptor, consequently obtaining some rotation invariance.

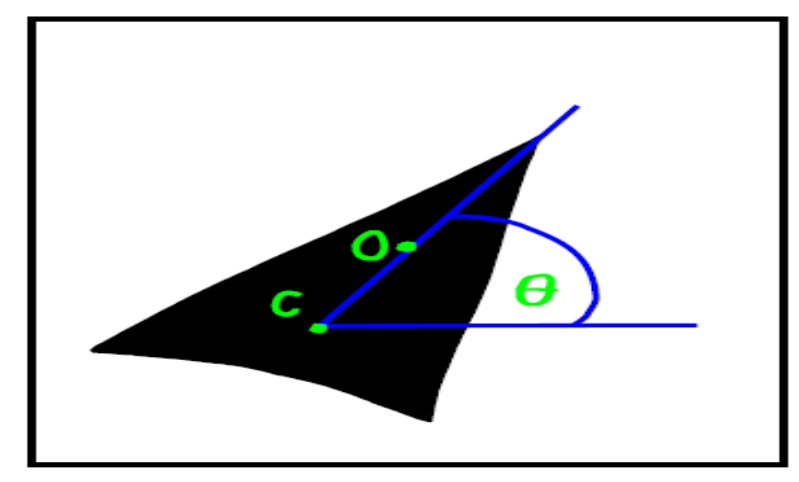

Figure 4. Rotation in a BRIEF algorithm

\subsection{Reasons to choose ORB algorithm}

Here are the status of Different Detector and descriptor:

SIFT:slow, good quality, not free,

SURF:fast + good quality, not free,

BRISK:fast + good quality, free

FREAK $\approx$ BRISK

ORB: faster + little bit less quality

\section{We found different comparison:}

Considering the above data as shown in Table 1, Table 2, and Table 3, we think ORB is the best algorithm to make faster and perfect Software for Face detection and Recognition [25-27]. So, we have chosen ORB as a detector and descriptor.

Table 1. Based on computation times for 1000 key points for diverse descriptors

\begin{tabular}{ccc}
\hline Descriptor & Run time $(\mathrm{ms})$ & Speed up() \\
\hline SURF & 117.1 & 3.83 \\
SIFT & 448.6 & 1.0 \\
BRIEF & 3.8 & 118.05 \\
BRISK & 10.6 & 42.32 \\
ORB & 4.2 & 106.80 \\
\hline
\end{tabular}

Table 2. Diverse descriptors average computation time

\begin{tabular}{ccc}
\hline Detector & Run time $(\mathrm{ms})$ & Speed up() \\
\hline SURF & 176 & 1.9 \\
FAST & 2 & 169 \\
BRISK & 10 & 33.8 \\
$O R B$ & 7 & 48.3 \\
\hline
\end{tabular}

Table 3. Speed-up over the sequential matching

\begin{tabular}{cccc}
\hline Descriptor & Size(bytes) & Run time $(\mathrm{ms})$ & Speed up \\
\hline SURF & 64 & 390 & 859.4 \\
SIFT & 128 & 2095 & 160.1 \\
BRIEF & 32 & 370 & 905.9 \\
BRISK & 64 & 524 & 640.1 \\
ORB & 32 & 370 & 905.9 \\
\hline
\end{tabular}

Indonesian J Elec Eng \& Comp Sci, Vol. 20, No. 2, November 2020 : 1078 - 1087 


\section{IMPLEMENTATION OF SYSTEM DESIGN}

\subsection{Flow chart}

In this process, the system detects face from a web camera and check with stored images, if the pattern matched then users allow login the system otherwise reject. This process is shown below like Figure 5:

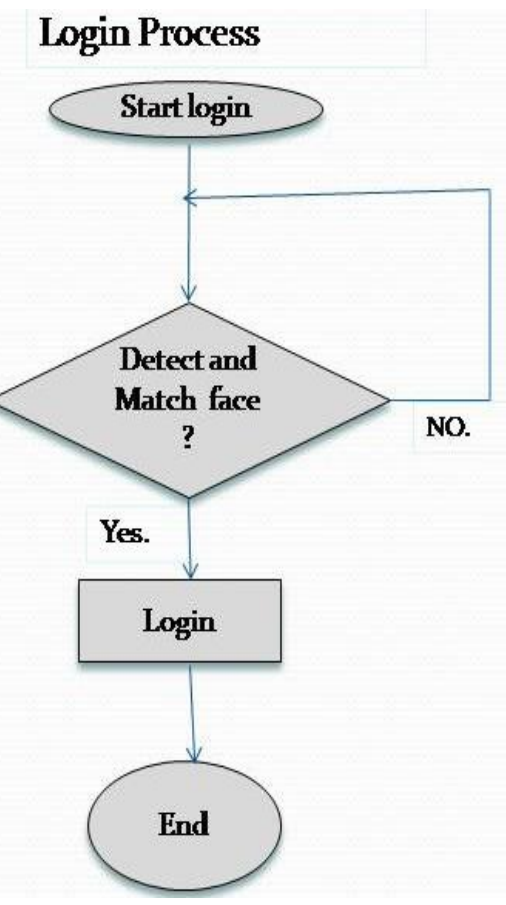

Figure 5. Flowchart of login system using face detection techniques

\subsection{Interface design}

If the system detects any face in front of the camera, it marks with a square and shows a captured face on the right side screen to save for further process as like as Figure 6 and Figure 7.

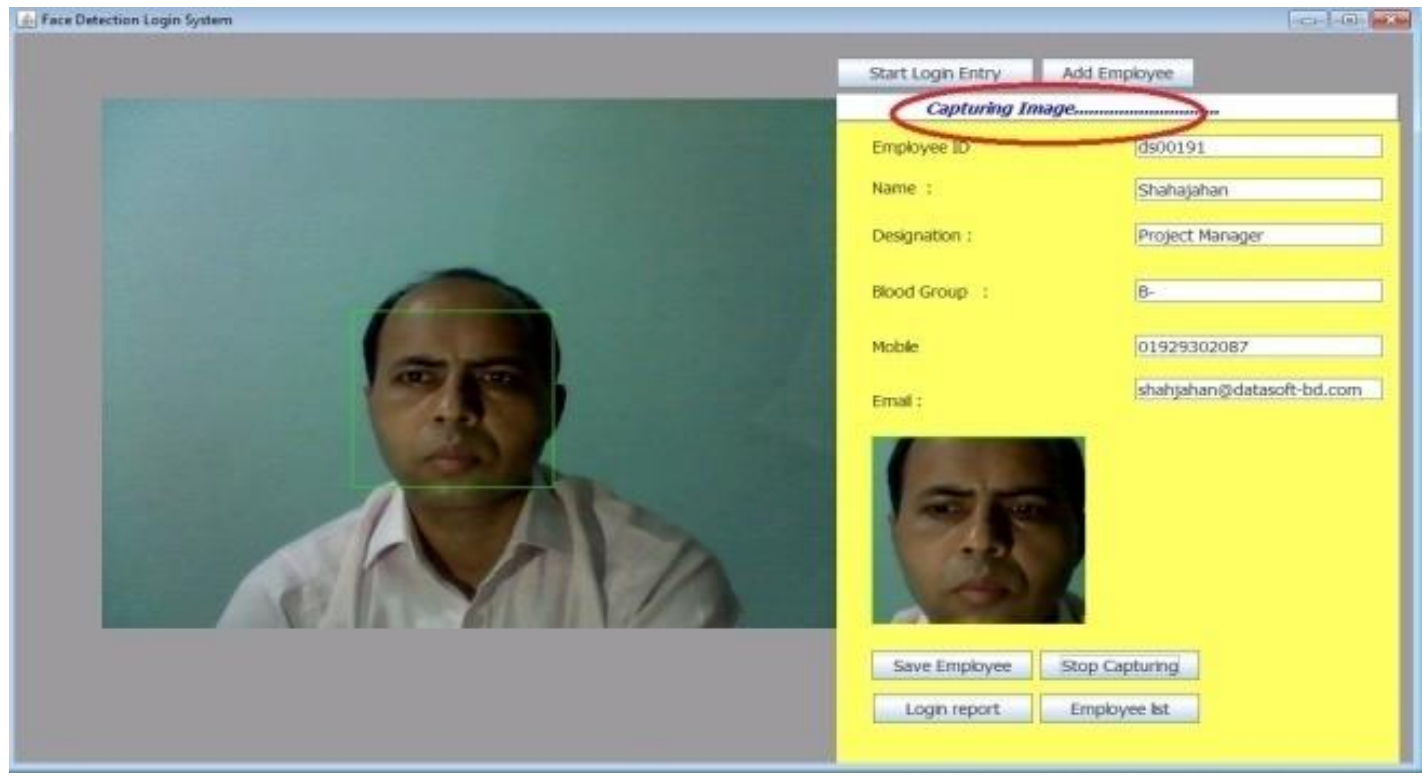

Figure 6. The interface of the system 


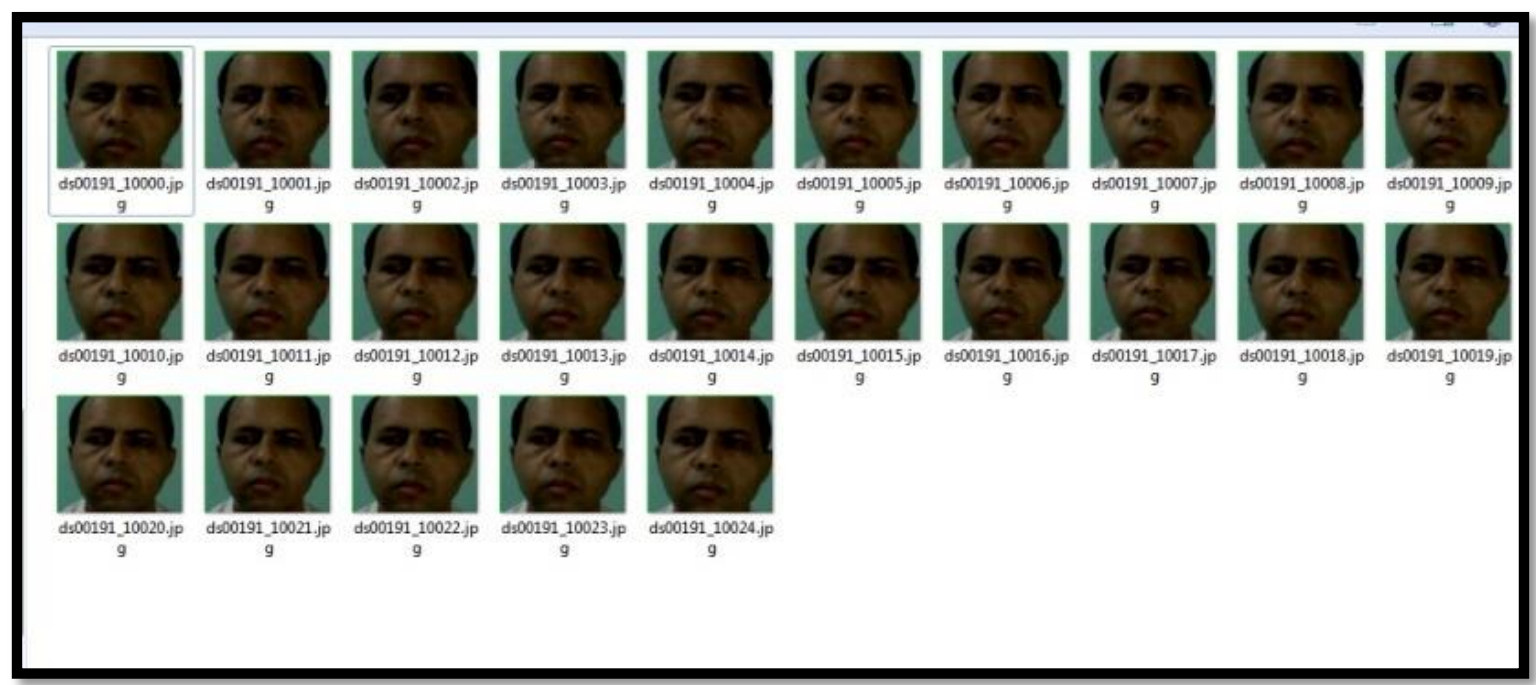

Figure 7. Sample of stored face images

Here, the user can add user details information with the image of the face. After saving user details information into the system, any user can $\log$ in from the next time. In this case, the user has to show his/her face in front of the camera, then the camera captures his/her image of the face and matches this image with the database images. If the image gets matched then it will show confirmation of login; otherwise the system will reject him/her and suggest trying again.

\section{RESULTS AND DISCUSSION}

To match the face with database, users' need to press the "Start Login Entry" button and put an Employee ID forwhich login he/she wants to do. Then keep the face of the employee in front of the camera. The system takes the image of the face through the camera and these images will then get matched with the database stored face images. If any image gets matched with any stored images then it collects all others stored images from the database and displays those on respective text fields and shows message login entry that has been done in the Figure 8 .

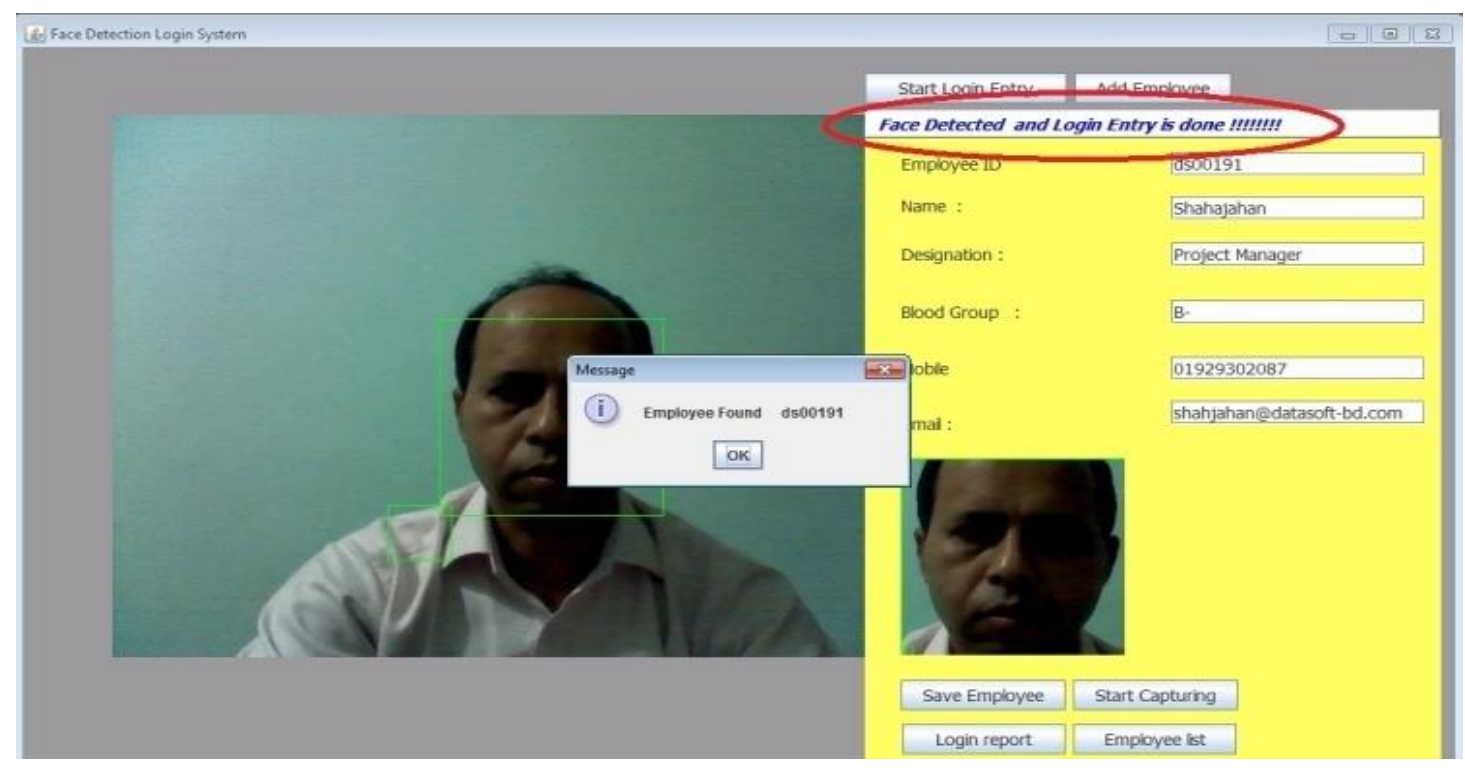

Figure 8. Valid Login entry 


\subsection{Performance analysis}

The performance of the system depends on the capacity of the server, camera and working environment. It takes 6-10 seconds on face recognition and 10-15 seconds for face matching or login on a Normal computer while it works with high performance on a high configured server such as HP 9 G server, here it takes 2-3 seconds on face detection and 4-5 seconds on the login system by matching the face with existing images.

\subsection{False rejection}

Sometimes when people try to login with a sweated face or bearded face, the system cannot recognize, in that case, it returns false rejection. Besides this, if people face change for various reasons such as sickness, hungriest, in that case, it does false rejection.

\subsection{False acceptance and matching thresh hold}

We have used ORB Algorithm for image matching with threshing hold for minimum feature matching. System checks features of face images, when the minimum matching distance becomes more than thresh hold 10 $(>10)$, it rejects and less than or equal to $(<=10)$, then it accepts as shown in Figure 9. From the system, a user can find the Login report and List of Employees as like as below Figure 10 and Figure 11.

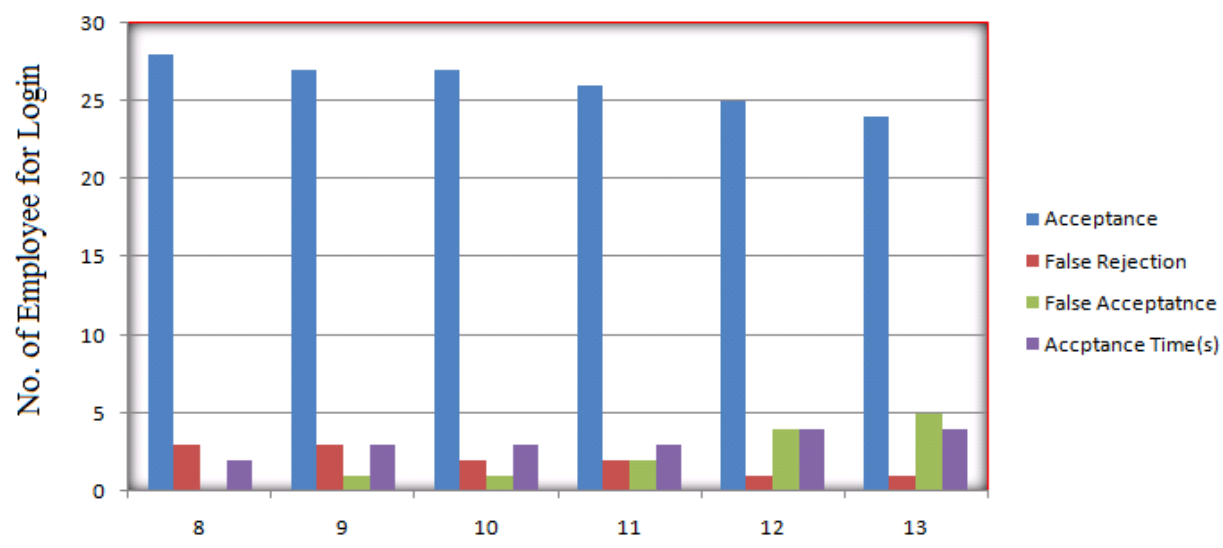

Minimum dist Matching Thresh hold.

Figure 9. The graph of acceptance, false rejection, false acceptance, and performance based on minimum dist. matching thresh hold

\section{Employee List}

\begin{tabular}{|c|c|c|c|c|c|}
\hline $\begin{array}{l}\text { Employee } \\
\text { Id }\end{array}$ & Name & Designation & $\begin{array}{l}\text { Blood } \\
\text { Group }\end{array}$ & Mobile No & E-mail id \\
\hline ds0010 & Mr. Alam & GM & $\mathrm{B}+$ & 01819225544 & malam@gmail.com \\
\hline ds00125 & Ms. Chy & DGM & A- & 01713548257 & chy212@yahoo.com \\
\hline ds00191 & Mr. Shahjahan & $\begin{array}{l}\text { Project } \\
\text { Manager }\end{array}$ & B- & 01929302087 & $\begin{array}{l}\text { shahjahan@datasoft- } \\
\text { bd.com }\end{array}$ \\
\hline ds00428 & $\begin{array}{l}\text { Mr.Md Hanif } \\
\text { Bhuyan }\end{array}$ & Asst DBA & $\mathrm{B}+$ & 01911434117 & $\begin{array}{l}\text { hanif.bhuy@datasoft- } \\
\text { bd.com }\end{array}$ \\
\hline ds00353 & $\begin{array}{c}\text { Mr.Shafiqul } \\
\text { Haider }\end{array}$ & Sr. Admin & $\mathrm{O}+$ & 01711702079 & faisal@gmail.com \\
\hline ds00396 & Mr.Shadat & $\begin{array}{l}\text { Software } \\
\text { Developer }\end{array}$ & O- & 01916688503 & shadat@yahoo.com \\
\hline ds00544 & $\begin{array}{l}\text { Mr.Anamul } \\
\text { Hoque }\end{array}$ & $\begin{array}{l}\text { Manager } \\
\text { Operations }\end{array}$ & $\mathrm{AB}+$ & 01819538742 & moin@ datasoft-bd.com \\
\hline ds00382 & Mr.Abdul Awal & $\begin{array}{l}\text { Software } \\
\text { Engineer }\end{array}$ & $\mathrm{O}+$ & 09238234 & $\begin{array}{l}\text { abdul.awal@datasoft- } \\
\text { bd.com }\end{array}$ \\
\hline ds00291 & $\begin{array}{l}\text { Ms.Khalan } \\
\text { Nandi }\end{array}$ & $\begin{array}{l}\text { Software } \\
\text { Engineer }\end{array}$ & $\mathrm{B}+$ & 01819525759 & khl.nan@gmail.com \\
\hline ds00271 & $\begin{array}{l}\text { Ms. Farjan } \\
\text { Boby }\end{array}$ & $\begin{array}{l}\text { Software } \\
\text { Developer }\end{array}$ & B- & 01717225588 & farjan.boby@gmail.com \\
\hline ds00127 & Ms.Dalia Fouzi & $\begin{array}{c}\text { Web } \\
\text { developer }\end{array}$ & O- & 01611336879 & dfozi@datasoft-bd.com \\
\hline
\end{tabular}

Figure 10. List of employees 


\begin{tabular}{|c|c|c|c|c|}
\hline \multirow{2}{*}{\multicolumn{5}{|c|}{ Print date: $10-F e b-2019$ 5:25 PM }} \\
\hline & & & & \\
\hline \multicolumn{5}{|c|}{ Date: 09-02-2019 } \\
\hline Emp Id & Name & Designation & $\begin{array}{l}\text { Login } \\
\text { Time }\end{array}$ & $\begin{array}{c}\text { Logout } \\
\text { Time }\end{array}$ \\
\hline ds00191 & Mr. Shahjahan & Project Manager & $8: 50$ am & $5: 15 \mathrm{pm}$ \\
\hline ds00428 & Mr.Md Hanif Bhuyan & Asst DBA & $8: 51$ am & $5: 15 \mathrm{pm}$ \\
\hline ds00125 & Ms. Chy & DGM & $8: 52$ am & 5:06 pm \\
\hline ds00353 & Mr.Shafiqul Haider & Sr. Admin & $8: 53$ am & 5:19 pm \\
\hline ds00010 & Mr. Alam & GM & $8: 55 \mathrm{am}$ & $5: 17 \mathrm{pm}$ \\
\hline ds00396 & Mr.Shadat & Software Developer & $8: 55$ am & $5: 10 \mathrm{pm}$ \\
\hline ds00291 & Ms.Khalan Nandi & Software Engineer & $8: 56$ am & $5: 16 \mathrm{pm}$ \\
\hline ds00271 & Ms.Farjan Boby & Software Developer & 8:57 am & $5: 15 \mathrm{pm}$ \\
\hline ds00544 & Mr.Anamul Hoque & $\begin{array}{l}\text { Manager } \\
\text { Operations }\end{array}$ & $8: 57$ am & $5: 18 \mathrm{pm}$ \\
\hline Ds00127 & Ms.Dalia Fouzi & Web developer & 8:58 am & 5:21 pm \\
\hline ds00382 & Mr.Abdul Awal & Software Engineer & $8: 59$ am & $5: 20 \mathrm{pm}$ \\
\hline \multicolumn{5}{|c|}{ Total 11} \\
\hline \multicolumn{5}{|c|}{ Date: 10-02-2019 } \\
\hline Emp Id & Name & Designation & $\begin{array}{l}\text { Login } \\
\text { Time }\end{array}$ & $\begin{array}{c}\text { Logout } \\
\text { Time }\end{array}$ \\
\hline ds00191 & Mr. Shahjahan & Project Manager & $8: 46$ am & $5: 20 \mathrm{pm}$ \\
\hline ds00428 & Mr.Md Hanif Bhuyan & Asst DBA & $8: 50$ am & 5:05 pm \\
\hline ds00353 & Mr.Shafiqul Haider & Sr. Admin & $8: 50$ am & $5: 06 \mathrm{pm}$ \\
\hline ds00125 & Ms. Chy & DGM & $8: 52$ am & $5: 06 \mathrm{pm}$ \\
\hline ds00271 & Ms.Farjan Boby & Software Developer & $8: 54$ am & $5: 13 \mathrm{pm}$ \\
\hline ds00544 & Mr.Anamul Hoque & $\begin{array}{l}\text { Manager } \\
\text { Operations }\end{array}$ & $8: 54$ am & $5: 14 \mathrm{pm}$ \\
\hline ds00396 & Mr.Shadat & Software Developer & $8: 55$ am & $5: 21 \mathrm{pm}$ \\
\hline ds00291 & Ms.Khalan Nandi & Software Engineer & $8: 56$ am & 5:07 pm \\
\hline ds00382 & Mr.Abdul Awal & Software Engineer & 9:00 am & $5: 21 \mathrm{pm}$ \\
\hline Ds00127 & Ms.Dalia Fouzi & Web developer & 9:00 am & 5:07 pm \\
\hline & & & \multicolumn{2}{|c|}{ Total 10} \\
\hline
\end{tabular}

Figure 11. Login report

\section{CONCLUSION}

In this research, we have developed a Smart Login Systems using Face Detection which is very efficient for any organization. We believe that organizations will be benefited by using this application because of easy access to this application. We have completed the research using different types of algorithms mostly the ORB algorithm to make it secure, faster and efficient. We have tested different users to make it more effective and error-free.

\section{REFERENCES}

[1] UsmanDastgeer, Hassam Nadeem,"Face Recognition - Lenovo VeriFace Login Application," [Online].Available:https://www.ida. liu .se/ T DDD17/oldprojects/2009/ projects/008

[2] YogeshManiktala, DurgeshSrivastava, BasantSah,"A robust technique of face recognition," International Journal of Advanced Research in Computer Science and Software Engineering. vol.6, no. 6, pp. 782-785, 2016.

[3] SmritiTikoo, Nitin Malik,"Detection of face using viola jones and recognition using back propagation neural network,"International Journal of Computer Science and Mobile Computing, vol. 5, no. 5, pp.288-295, 2016.

[4] Nita Thakare, MeghnaShrivastava, NidhiKumari,NehaKumari, Darleen Kaur, RinkuSingh, "Face detection and recognition for automatic attendance system,"International Journal of Computer Science and Mobile Computing, vol. 5, no. 4, pp.74-78, 2016.

[5] Gunjan Mehta, Sonia Vatta, "An introduction to a face recognition system using PCA, FLDA and artificial neural networks,"International Journal of Advanced Research in Computer Science and Software Engineering, vol. 3, no. 5. pp. 1418-1420, 2013.

[6] M.Nandini,P.Bhargavi,G.RajaSekhar, "Face recognition using neural networks,"International Journal of Scientific and Research Publications, vol 3, no. 3, 2013

[7] Mamata. Kalas, "Real time face detection and tracking using OpenCV," International Journal of Soft Computing and Artificial Intelligence, vol.2, no. 1, pp. 41-44, 2014.

[8] D. A. R. Wati and D. Abadianto, "Design of face detection and recognition system for smart home security application," 2nd International conferences on Information Technology, Information Systems and Electrical Engineering (ICITISEE), Yogyakarta, pp. 342-347, 2017.

[9] S Aanjana Devi, V Palanisamy, R AnandhaJothi, "Confidential e-voting system using face detection and recognition,"International Journal of Engineering and Techniques, vol 3, no. 4, pp. 81-85, 2017. 
[10] Hapsari, Gita Indah, GivaAndrianaMutiara, and HuseinTarigan, "Face recognition smart cane using haar-like features and eigenfaces," TELKOMNIKA (Telecommunication Computing Electronics and Control), vol. 17, no. 2, pp. 973-980, 2019.

[11] Wibowo, Hardianto, et al., "Facial expression recognition of 3D image using facial action coding system (FACS)," TELKOMNIKA (Telecommunication Computing Electronics and Control), vol. 17, no. 2, pp.628-636, 2019.

[12] Persada, Reivind P., and SuciAulia."Automatic face and VLP'S recognition for smart parking system," TELKOMNIKA (Telecommunication Computing Electronics and Control), vol. 17, no. 4, pp. 1698-1705,2019.

[13] Ghazal, Mohammed Talal, et al., "Face recognition based on curvelets, invariant moments features and SVM." TELKOMNIKA (Telecommunication Computing Electronics and Control), vol. 18, no. 2, pp. 733-739, 2020.

[14] J. Vinoj, S. Gavaskar, G. P. Rameshkumar, R. Priya, "Smart city's vehicle login system with sensor chip," IEEE International Conference on Advances in Computer Applications (ICACA),pp. 217-223, 2016.

[15] Kumar, Ashu, AmandeepKaur, and Munish Kumar, "Face detection techniques: A review," Artificial Intelligence Review, vol.52, no. 2, pp. 927-948, 2019.

[16] Ali, Abdelmgeid A., TarekAbd El-Hafeez, and YosraKhalafMohany, "An accurate system for face detection and recognition, "Journal of Advances in Mathematics and Computer Science, pp. 1-19, 2019.

[17] $\mathrm{Li}$, Chun-Ta, et al. "An efficient user authentication and user anonymity scheme with provably security for IoTbased medical care system," Sensors, vol. 17, no. 7,pp. 1482, 2017.

[18] Bhatti, Kaneez, et al., "Smart attendance management system using face recognition," EAI Endorsed Transactions on Creative Technologies, vol. 5, no. 17, 2018.

[19] Sakshi Patel, Prateek Kumar, Shelesh Garg, Ravi Kumar, "Face Recognition based smart attendance system using IoT,", International Journal of Computer Sciences and Engineering, vol. 5, pp. 871- 877, 2018.

[20] Akbar, MdSajid, et al., "Face recognition and RFID verified attendance system," International Conference on Computing, Electronics \& Communications Engineering (iCCECE), IEEE, 2018.

[21] Arsenovic, Marko, et al., "FaceTime-Deep learning based face recognition attendance system,"15th International Symposium on Intelligent Systems and Informatics (SISY), 2017.

[22] [Online]. Available: https://opencv-python-tutroals.readthedocs.io/en/latest/py_tutorials/py_feature2d/py_orb/py_orb.html\#orb

[23] Rosten, Edward, and Tom Drummond. "Machine learning for high-speed corner detection." European conference on computer vision. Springer, Berlin, Heidelberg, 2006.

[24] [Online]. Available: https://readthedocs.org/projects/opencv-python-tutroals/downloads/pdf/latest/

[25] Fredrik Walterson and KenanBabic,"Face identifcation in near IR-videos using Smart Eye tracking profles," Chalmers University of Technology Gothenburg, Sweden, pp.15-19, 2014

[26] Stefan Haller,"An update on features on SURF, BRISK, ORB and FREAK" Technische University, Dresden, 2015

[27] Shaharyar Ahmed Khan Tareen, Zahra Saleem, "A Comparative Analysis of SIFT, SURF, KAZE, AKAZE, ORB, and BRISK,"International Conference on Computing, Mathematics and Engineering Technologies, pp. 1-10, 2018.

\section{BIOGRAPHIES OF AUTHORS}
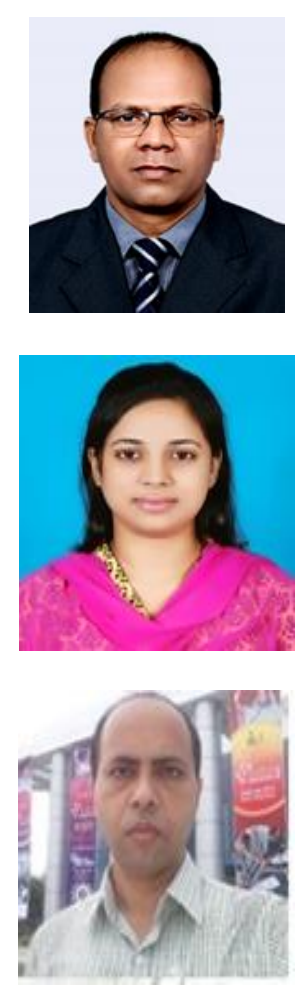

Mohammad JahangirAlam has completed post-graduation (MSc in CSIT) in 2008 from Southern University Bangladesh, Chittagong. He is an Assistant Professor and Coordinator at the department of Computer Science and Information Technology in Southern University Bangladesh, Chittagong. He has published more than 20 technical research papers in reputed national, International Journals and Conferences. His research interest includes Cloud Computing, Mobile apps design, Health Informatics, Bid Data analytics etc.

Tanjia Chowdhury is working as a Lecturer in the Department of Computer Science and Information Technology at Southern University Bangladesh, Chittagong, Bangladesh. She has received her B.Sc. and M. Sc. Engineering from University of Chittagong, Chittagong. She has published two books in Germany and five research papers in different international journals. Her area of interest is Networking, Artificial Intelligence.

Md. Shahzahan Ali has completed his post-graduation (MSc in CSIT) in 2018 from Southern University Bangladesh, Chittagong. Currently he is working as a Manager at Chittagong Container Terminal Management System (CTMS) project in Datasoft Systems Bangladesh Ltd and has developed several modules of the software being used in CTMS and supports all kinds of software solution as per business needs.

\footnotetext{
A smart login system using face detection and recognition by ORB algorithm (Mohammad Jahangir Alam)
} 